University of Texas at El Paso

ScholarWorks@UTEP

$5-2002$

\title{
Fuzzy Measures and Integrals as Aggregation Operators: Solving the Commensurability Problem
}

Francois Modave

Vladik Kreinovich

The University of Texas at El Paso, vladik@utep.edu

Follow this and additional works at: https://scholarworks.utep.edu/cs_techrep

Part of the Computer Engineering Commons

Comments:

UTEP-CS-02-15.

Published in the Proceedings of the 21st International Conference of the North American Fuzzy Information Processing Society NAFIPS'2002, New Orleans, Louisiana, June 27-29, 2002, pp. 292-297.

\section{Recommended Citation}

Modave, Francois and Kreinovich, Vladik, "Fuzzy Measures and Integrals as Aggregation Operators:

Solving the Commensurability Problem" (2002). Departmental Technical Reports (CS). 345.

https://scholarworks.utep.edu/cs_techrep/345

This Article is brought to you for free and open access by the Computer Science at ScholarWorks@UTEP. It has been accepted for inclusion in Departmental Technical Reports (CS) by an authorized administrator of ScholarWorks@UTEP. For more information, please contact Iweber@utep.edu. 


\title{
Fuzzy Measures and Integrals as Aggregation Operators: Solving the Commensurability Problem
}

\author{
François Modave and Vladik Kreinovich \\ CS Dept. University of Texas at El Paso, \\ $500 \mathrm{~W}$. University Ave. \\ El Paso, Tx, 79968-0518 \\ email: \{fmodave, vladik \}@cs.utep.edu
}

\begin{abstract}
The aim of this paper is to shed some light on the use of fuzzy measures and integrals as aggregation operators in multicriteria decision making. These techniques have been widely used on an ad hoc basis, but with no axiomatization. It is possible to obtain preference representation theorems in multicriteria decision making problems, relying on a formal parallelism between decision under uncertainty and multicriteria decision making. Though, it raises some commensurability problems. In this paper, we show how to obtain an axiomatization of multicriteria decision making problems, in a very natural way, and we show how to solve the commensurability problem in a particular case.
\end{abstract}

\section{Introduction}

In decision theory, we aim at representing the preferences of a decision maker, but also at understanding the decision maker's behavior. This pertains to both decision under uncertainty and multicriteria decision making.

Let us denote (in the uncertainty framework) by $S$ the set of states of the world, and by $X$ the set of consequences. Generally, the decision maker has clear preferences over $X$, but the preferences over the actions she/he will take to lead to some consequences depend on the true state of the world which is a priori unknown. Therefore, decision under uncertainty aims at "translating" the preferences over $X$ into a preference relation $\succeq$ over the actions. A simple example is a decision maker investing money in the Stock market. The decision maker will exhibit clear preferences over the consequences (e.g. earning money for a rational decision maker). But, the problem is actually to decide whether to sell or buy, or do nothing, with no a priori knowledge of the true state of the world (the evolution of the Stock market).

Thanks to the work of Savage, we know that under a set of hypotheses over $S, X$ and $\succeq$, there exists a unique probability measure $P$ over $S$ and a unique (up to an affine transformation) utility function $u$ : $X \rightarrow \mathbb{R}$ such that, if $f$ and $g$ are two actions:

$$
f \succeq g \Leftrightarrow \int_{S} u(f(s)) d P(s) \geq \int_{S} u(g(s)) d P(s) .
$$

When examining the properties over $\succeq$ we quickly realize that they will not be fulfilled in practice and therefore need to be relaxed. When doing so, we need to drop the independence hypothesis of Savage, namely the sure-thing principle. This was done by Schmeidler [14], who obtained a similar result to the one of Savage where the probability measure is replaced by a more general measure which is only monotonic with respect to the inclusion.

In the multicriteria decision making paradigm, where the decision maker has to express preferences over a multidimensional set $X=X_{1} \times \cdots \times X_{n}$. The decision maker is able to express preferences over a set $X_{i}$, but needs to find a way to "aggregate" these partial preferences into a global preference. For example, a customer wants to buy a car that belongs to a set $X$ (therefore the car is represented by its attributes or criteria). The decision maker has clear preferences over a set of values of attributes $X_{i}$ (e.g. $200 \mathrm{hp}$ is preferred to $150 \mathrm{hp}$ ), but has no such clear preferences between two cars, due to compensatory effects between values of attributes. 
Fuzzy measures and integrals have been widely used as aggregation operators. Nonetheless, there was no axiomatization as was the case in decision under uncertainty.

One of the authors of this paper proposed to use a formal parallelism between multicriteria decision making and decision under uncertainty to use the already existing representation results of decision under uncertainty to generate in a very natural way similar representation results in multicriteria decision making with respect to some fuzzy measure and a fuzzy integral associated to this fuzzy measure, an axiomatization that was lacking so far. When doing some, commensurability hypothesis between the sets $X_{i}$ 's has to be introduced but was not very satisfactory so far.

The present paper aims at presenting the above transformation process as well as solving the commensurability problem under a weak hypothesis. In the first section of this paper, we briefly recall basics of fuzzy integration, then present them from a decision making point of view. We show under which conditions, decision under uncertainty and multicriteria decision making are equivalent. This leads us very naturally to the commensurability problem, for which we present a solution.

\section{Fuzzy integration and decision theory}

In the sequel, we will restrict ourselves to the finite case, for the sake of simplicity. Besides, to establish a parallelism between uncertainty and multicriteria decision making, this is necessary as we will see.

\subsection{Fuzzy integration theory}

In this section, $\Omega$ is a finite set and $\mathcal{P}(\Omega)$ is the set of subsets of $\Omega$. We briefly recall the definitions of fuzzy measures and Choquet integral (for more details see for example [2], [1]).

Definition 1 A fuzzy measure (or non-additive measure) on $(\Omega, \mathcal{P}(\Omega))$ is a set function $\mu: \mathcal{P}(\Omega) \rightarrow$ $[0,1]$ such that

(1) $\mu(\emptyset)=0, \mu(X)=1$,

(2) if $A, B \in \mathcal{P}(\Omega), A \subset B$, then $\mu(A) \leq \mu(B)$, that is, $\mu$ is a non decreasing set function w.r.t inclusion.

Remark: Fuzzy measures are also called nonadditive measures, capacities...

We will note that a probability measure is a fuzzy measure as the addivity of the probability implies the monotonicity property.

Definition 2 Let $\mu$ be a fuzzy measure on $(\Omega, \mathcal{P}(\Omega))$ and an application $f: \Omega \rightarrow[0,+\infty]$. The Choquet integral of $f$ w.r.t $\mu$ is defined by:

$$
\text { (C) } \int_{\Omega} f d \mu=\sum_{i=1}^{n}\left(f\left(\omega_{(i)}\right)-f\left(\omega_{(i-1)}\right)\right) \mu\left(A_{(i)}\right)
$$

where the subscript (.) indicates that the indices have been permuted in order to have $f\left(\omega_{(1)}\right) \leq \cdots \leq$ $f\left(\omega_{(n)}\right), A_{(i)}=\left\{\omega_{(i)}, \ldots, \omega_{(n)}\right\}$ and $f\left(\omega_{(0)}\right)=0$, by convention.

It is easy to see that the Choquet integral is a classical Lebesgue integral up to a reordering of the indices. Besides, if the fuzzy measure $\mu$ is additive, then the Choquet integral reduces to a Lebesgue integral. We refer the reader to [8] for properties of fuzzy measures, and properties of the Choquet integral.

\subsection{Applications to decision making}

Let us consider a decision problem under uncertainty $(S, X, A, \succeq)$. We know that additive representations are not sufficient to model every facet of human behavior and therefore a non-additive representation is necessary. Schmeidler [14], [15] has proved that under a set of conditions on the preference relation $\succeq$ (continuity and independence in some sense), there exists a Choquet representation of the preference relation, that is, there exist a function $u: X \rightarrow \mathbb{R}$, unique up to an affinity, and a unique fuzzy measure $\mu$ on $S$ such that: for all acts $f, g \in A$ we have:

$$
f \succeq g \Leftrightarrow(C) \int_{S} u(f(\cdot)) d \mu \geq(C) \int_{S} u(g(\cdot)) d \mu .
$$

This result has been refined later by Gilboa [7], then by Wakker [17]. In the multicriteria framework, let us assume we have a cartesian product set 
$X=X_{1} \times \cdots \times X_{n}$, with a preference relation $\succeq$ on $X$. Let $u_{i}: X_{i} \rightarrow \mathbb{R}$ be the i-th monodimensional utility function. Then, we need to find an aggregation operator $\mathcal{H}: \mathbb{R}^{n} \rightarrow \mathbb{R}$ such that for all $x=\left(x_{1}, \cdots, x_{n}\right)$ and $y=\left(y_{1}, \cdots, y_{n}\right)$ we have:

$$
\begin{aligned}
x \succeq y \Leftrightarrow & \mathcal{H}\left(u_{1}\left(x_{1}\right), \cdots, u_{n}\left(x_{n}\right)\right) \geq \\
& \mathcal{H}\left(u_{1}\left(y_{1}\right), \cdots, u_{n}\left(y_{n}\right)\right) .
\end{aligned}
$$

It is known that many aggregation usual operators can be written as a Choquet integral, that is, there exists a fuzzy measure $\mu$ on the set of criteria $I=\{1, \cdots, n\}$ such that $\mathcal{H}\left(u_{1}\left(x_{1}\right), \cdots, u_{n}\left(x_{n}\right)\right)=$ $\sum_{i=1}^{n}\left[\left(u_{(i)}\left(x_{(i)}\right)-u_{(i-1)}\left(x_{(i-1)}\right)\right] \mu\left(A_{(i)}\right)\right.$ with the same notations as before (see [8]).

A very natural choice is to put weights $\alpha_{1}, \cdots, \alpha_{n} \in$ $[0,1]$, such that $\sum_{i} \alpha_{i}=1$, that represents the importance given to each criterion, by the decision maker and to choose the aggregation operator

$$
\mathcal{H}\left(u_{1}\left(x_{1}\right), \cdots, u_{n}\left(x_{n}\right)\right)=\sum_{i} \alpha_{i} u_{i}\left(x_{i}\right)
$$

That is, the global score of an alternative is simply a weighted sum of the partial score.

Unfortunately, this strategy (or any additive strategy) is not sufficient to represent dependencies between criteria or attributes as is shown in the measurement theory point of view in [9], or in [10].

Strangely enough, the reason why additivity is not sufficient in the MCDM perspective is similar to the equivalence between the sure-thing principle and the existence of a probability measure in decision under uncertainty. We have shown in [3] that the surething principle and the principle allowing an additive aggregation operator, namely the mutual preferential independence of criteria

Definition 3 Let $X=X_{1} \times \cdots \times X_{n}$ a set with a preference relation $\succeq$. A subset $J$ of $I=\{1, \cdots, n\}$ is said to be preferentially independent of $J^{c}$ iff for every $x_{J}, y_{J} \in X_{J}$, for every $x_{J^{c}}, z_{J^{c}} \in X_{J^{c}}$ we have

$$
\left(x_{J}, x_{J^{c}}\right) \succeq\left(y_{J}, x_{J^{c}}\right) \Leftrightarrow\left(x_{J}, z_{J^{c}}\right) \succeq\left(y_{J}, z_{J^{c}}\right)
$$

where $X_{J}=\times_{i \in J} X_{i}$ and $x_{J}$ is an element of $X_{J}$. If this property holds for every subset of $I$, we say that $\succeq$ verifies the mutual preferential hypothesis.

are formally equivalent when the set of states of the world $S$ is finite. Therefore, the fact that decision under uncertainty and multicriteria decision making are formally equivalent (up to some technical details) should not come as a surprise.

Fuzzy measures and integrals, which are nonadditive operators, become natural candidates to avoid the drawbacks of additive methods. In this case, the fuzzy measure is defined on the criteria, and acts like a weighting operator, that is, an operator defining the importance of a coalition of criteria. This is not the only interpretation of fuzzy measures in multicriteria decision making. Indeed, fuzzy measures can be used to define the interaction between two criteria $i, j \in I$ in the following way [13]:

$$
\begin{gathered}
I_{i j}=\sum_{K \subset X \backslash\{i, j\}} \xi(|K|)[\mu(K \cup\{i, j\}) \\
-\mu(K \cup i)-\mu(K \cup j)+\mu(\{i, j\})]
\end{gathered}
$$

with $\xi(k)=\frac{(n-k-2) ! k !}{(n-1) !}$. This representation through interaction indices happens to be much closer to the decision maker's mind than the usual measure representation.

\section{Uncertainty and Multicriteria are Equivalent}

\subsection{From (DU) to (MCDM)}

As the authors have shown in [4] and as it was already noticed before in [6] for instance, decision under uncertainty with a finite number of states of the world and multicriteria decision making are formally equivalent. Indeed, let $f$ be an act of an uncertainty decision problem with a finite number of states and $S=\left\{s_{1}, \cdots, s_{n}\right\}$. Let $f\left(s_{1}\right), \cdots, f\left(s_{n}\right)$ be the values of $f$ in $X$. Then each act can be identify with a vector of $X^{n}$. Therefore the comparison of two acts $f$ and $g$ reduces to compare their values:

$$
f \succeq g \Leftrightarrow\left(f\left(s_{1}\right), \cdots, f\left(s_{n}\right)\right) \succeq\left(g\left(s_{1}\right), \cdots, g\left(s_{n}\right)\right) .
$$

Compared to the multicriteria decision notation:

$$
x \succeq y \Leftrightarrow\left(x_{1}, \cdots, x_{n}\right) \succeq\left(y_{1}, \cdots, y_{n}\right)
$$

we see that the problem of decision under uncertainty has been written in a multicriteria decision framework by identifying the states of the world with the criteria and the acts with the consequences. 


\subsection{From (MCDM) to (DU)}

Actually, the above allows us to write a decision under uncertainty problem as a multicriteria decision making problem, where the uncertainty on the states of the world becomes equivalent to identifying the most important criterion, or set of criteria. Nonetheless, it does not allow us to write a multicriteria decision making problem as a problem under uncertainty as the set of alternatives in multicriteria decision making setting is $X_{1} \times \cdots \times X_{n}$ where the sets $X_{i}$ can be different, whereas in uncertainty the set of consequences is $X^{n}$. Therefore, we need to find a way to send the sets $X_{i}$ into a common scale.

The solution to this problem is actually very simple and lies in the foundations of measurement theory (see for example [9] for a thorough presentation) that aims at finding necessary and/or sufficient conditions for the existence of order-preserving isomorphisms between ordered-algebraic structures and "more understandable" sets (e.g. the set of real numbers endowed with its natural order $(\mathbb{R}, \geq)$.

In the sequel, wwe assume that each set $X_{i}$ is endowed with a weak order $\succeq_{i}$ that is a binary relation satisfying

(1) $\forall x_{i}, y_{i} \in X_{i}$, either $x_{i} \succeq_{i} y_{i}$ or $y_{i} \succeq_{i} x_{i}$ (connectedness)

(2) $\forall x_{i} \in X_{i}, x_{i} \succeq_{i} x_{i}$ (reflexivity)

(3) $\forall x_{i}, y_{i}, z_{i} \in X_{i}, x_{i} \succeq_{i} y_{i}$ and $y_{i} \succeq_{i} z_{i}$ implies $x_{i} \succeq_{i} z_{i}$ (transitivity)

Remark: We can assume that the weak order is an order that is $x \succeq y$ and $y \succeq x$ implies $x=y$ by using the quotient set $X / \sim$.

Our first step is to construct a function $\phi_{i}: X_{i} \rightarrow \mathbb{R}$ such that $x_{i} \succeq_{i} y_{i} \Leftrightarrow \phi_{i}\left(x_{i}\right) \geq \phi_{i}\left(y_{i}\right)$ that is an order-preserving function from $X_{i}$ to the real numbers. Under what conditions can we construct such a function? The idea is very simple. We know that the set $\mathbb{Q}$ of rational numbers is dense in $\mathbb{R}$ that is, between two different real numbers, we can find a rational number. Besides, $\mathbb{Q}$ is at most countable. The answer to our problem is simply to copy this property of the real numbers, called separability.

Definition 4 Let $(X, \succeq)$ be a set with a weak order relation. A subset of $X, A$ is said to be order-dense w.r.t $\succeq$ iff for all $x, y \in X, x \succ y$, there exists $a \in A$ such that $x \succeq a \succeq y . X$ is said to be order-separable iff there exists an order-dense, at most countable subset of $X$.

Then, we can prove the following:

Then, we have the following theorem:

Theorem 1 Let $(X, \succeq)$ be a set with a weak order. Then there exists an application $\phi: X \longrightarrow \mathbb{R}$ such that $\forall x, y \in X$ the following propositions are equivalent

(1) $x \succeq y \Leftrightarrow \phi(x) \geq \phi(y)$

(2) $X$ is order-separable.

If $\psi$ is an other function verifying (1) and (2) then there exists a non-decreasing bijection $f: \mathbb{R} \rightarrow \mathbb{R}$ such that $\psi=f \circ \phi$.

(see [9] for a proof).

Therefore if we assume that each set $X_{i}$ is orderseparable for its weak order, we have our transformations to a common scale $\mathbb{R}$. Note that from an application point of view, this is not restrictive as any finite, or at most countable set if order-separable.

These functions allow us now to transform the sets $X_{i}$ in a common scale, and to write any multicriteria decision making problem as a decision under uncertainty problem. With some additional restrictions, we can show how we can use the representation theorems derived in the uncertainty paradigm, to prove similar results in the multicriteria paradigm as was shown in [11] following Wakker, and [12] for an extension to lotteries.

\section{The Commensurability problem}

In this section, we consider a multicriteria decision making problem $\left(X=X_{1} \cdots \times \cdots X_{n}, \succeq\right)$ where each set $X_{i}$ is endowed with a weak order $\succeq_{i}$, and is an order-separable set, i.e. for each $i=1, \cdots, n$ there exists a set $A_{i}$ in $X_{i}$ which is order-dense and at most countable. Then, we know from the previous section that for each $i=1, \cdots, n$ there exits an application $\phi_{i}: X_{i} \rightarrow \mathbb{R}$ such that

$$
x_{i} \succeq_{i} y_{i} \Leftrightarrow \phi_{i}\left(x_{i}\right) \geq \phi_{i}\left(y_{i}\right) .
$$


Now, consider the following problem. Assume that $i$ and $j$ are different and let us take $x_{i}$ and $x_{j}$ in $X_{i}$ and $X_{j}$ respectively. Because the functions $\phi_{i}$ and $\phi_{j}$ are constructed independently, it can happen that

$$
\phi_{i}\left(x_{i}\right)=\phi_{j}\left(x_{j}\right)
$$

Does it entail that $x_{i}$ and $x_{j}$ are equivalent in some sense? Generally no.

We will restrict the commensurability problem, to the following case: we assume that there exists an equivalence relation $\sim$ between some elements

$$
z \in Z \subset \cup_{i=1}^{n} X_{i}
$$

that is, the decision maker is able to express to indifference between elements in different sets. We have a partial weak order over $\cup_{i=1}^{n} X_{i}$.

An example of where such a situation could arise is if a customer wants to buy a car and has the choice between two cars, one with a very good consumer report on security, the other with a very low gas consumption. We assume the customer has no clear preference between these two alternatives.

Now, the functions $\phi_{i}$ we have constructed are not unique. So our question is, can we construct such a family, order-preserving for each $X_{i}$, that will also represent the weak order over $Z$. We will assume that $Z$ with this weak order is order-separable and denote by $A$ an order-dense subset of $Z$ which is at most countable and $a_{j}$ will denote an element of $A$.

We will now show that such a selection if possible by constructing a function $\phi: X \rightarrow \mathbb{R}$ which is orderpreserving over $Z$ and is derived from the functions $\phi_{i}$. For the sake of clarity, we will write $\phi$ with no index for $\phi\left(a_{j}\right)$ as each $a_{j}$ can be in a set $X_{1}$ or $X_{4}$ etc...

For every $k$ let us define the following:

$$
\phi^{-}=\max \left\{\phi\left(a_{j}\right) \mid a_{k} \succ a_{j}, k \geq j\right\}
$$

and similarly,

$$
\phi^{+}=\min \left\{\phi\left(a_{j}\right) \mid a_{j} \succ a_{k}, j \geq k\right\}
$$

Now, if both $\phi^{-}$and $\phi^{+}$are finite for a given $k$, the value of the constructed function at $a_{k}$ is the midpoint between $\phi^{-}$and $\phi^{+}$. Else, we will choose $\phi^{-}+1$ if $\phi^{+}$is infinite, or $\phi^{+}-1$ if $\phi^{-}$is infinite.
Then, for every element $z \in Z$, we define the orderpreserving function $\phi$ by

$$
\phi(x)=\sup \{\phi(a) \mid x \succ a\}
$$

From this construction, it is clear that we have

$$
x \sim y \Leftrightarrow \phi(x) \geq \phi(y)
$$

And therefore, we have the existence of an orderpreserving family of functions, accomodating for the partial weak order.

\section{Conclusion}

In this paper, we have presented how fuzzy measures and integrals were a natural choice to replace the probabilistic approach traditionally used in decision under uncertainty. Then, we have shown that decision under uncertainty and multicriteria decision making were formally equivalent under the conditions that the set of states of the world are finite, and the order-separability of the sets of values of attributes. This led us to a commensurability problem. We have seen how this problem could be solved if we assumed the set $\cup_{i=1}^{n} X_{i}$ to be endowed with a partial weak order, and order-separable. Then, a previous paper has shown how to use the results existing in decision under uncertainty to derive representation theorems in multicriteria decision making, offering a simple axiomatization of the field, at least with the Choquet integral. A next step is to provide a similar approach in a more qualitative setting, using the Sugeno integral [16] following the axiomatization proposed in [5].

\section{References}

[1] G. Choquet. Theory of capacities. Annales de l'Institut Fourier, 5, 1953.

[2] D. Denneberg. Non-Additive Measure and Integral. Kluwer Academic Publisher, 1994.

[3] D. Dubois, M. Grabisch, F. Modave, and H. Prade. Relating decision under uncertainty and MCDM models: an AI perspective. In $I J$ CAI, Nagoya, Japan, August 1997. poster.

[4] D. Dubois, M. Grabisch, F. Modave, and H. Prade. Un parallèle entre décision dans 
l'incertain et décision multicritère dans une perspective IA. In Actes de conf. Rep. Formes et Intel. Art., Clermont-Ferrand, Janvier 1998.

[5] D. Dubois, H. Prade, and R. Sabbadin. A possibilistic logic machinery for qualitative decision. In AAAI Workshop, Stanford, Ca, March 1997.

[6] P. Fishburn and P. Wakker. The invention of the independance condition of preferences. Management Science, 41:1130-1144, 1995.

[7] I. Gilboa. Expected utility with purely subective non-additive probabilities. J. of Math. Economics, 16(1):65-88, 1987.

[8] M. Grabisch, H. T. Nguyen, and E. A. Walker. Fundamentals of Uncertainty Calculi with Applications to Fuzzy Inference. Kluwer Academic Publisher, Dordrecht, 1995.

[9] D. Krantz, R. Luce, P. Suppes, and A. Tverski. Foundations of Measurement. Academic Press, 1971.

[10] F. Modave and M. Grabisch. Preferential independence and the Choquet integral. In 8th Int. Conf. on the Foundations and Applicatons of Decision under Risk and Uncertainty (FUR), Mons, Belgium, July 1997.

[11] F. Modave and M. Grabisch. Preference representation by the Choquet integral: the commensurability hypothesis. In Proc. 7th Int. Conf. on Information Processing and Management of Uncertainty in Knowledge-Based Systems (IPMU), Paris, France, July 1998.

[12] F. Modave and M. Grabisch. Lotteries in multicriteria decision making framework. In Proc. 8th Int. Conf. on Information Processing and Management of Uncertainty in KnowledgeBased Systems (IPMU), Madrid, Spain, July 2000.

[13] T. Murofushi and S. Soneda. Techniques for reading fuzzy measures (iii): interaction index. In Proc. 9th Fuzzy System Symposium, pages 693-696, Sapporo, Japan, May 1993. In Japanese.

[14] D. Schmeidler. Integral representation without additivity. Proc. of the Amer. Math. Soc., 97(2):255-261, 1986.
[15] D. Schmeidler. Subjective probability and expected utility without additivity. Econometrica, 57(3):571-587, 1989.

[16] M. Sugeno. Theory of fuzzy integrals and its applications. $\mathrm{PhD}$ thesis, Tokyo Inst. of Technology, 1974.

[17] P. Wakker. A behavioral foundation for fuzzy measures. Fuzzy Sets \& Systems, 37:327-350, 1990. 